\title{
AN ATOMIC DECOMPOSITION OF CONTINUA INTO APOSYNDETIC CONTINUA
}

\author{
BY \\ LOUIS F. MCAULEY
}

Introduction. Perhaps some of the most interesting problems in mathematics are concerned with the definability of specific types of continuous real-valued functions on a compact set $M$. Among important contributions to solutions of these problems are many generalizations. Both R. L. Moore $[5 ; 6]$ and G. T. Whyburn $[7 ; 8 ; 9]$ have described continuous transformations of a compact metric continuous curve onto an acyclic continuous curve whose inverse sets are continua (i.e., monotone transformations).

In a recent paper [4], the author generalized methods of Moore and Whyburn by which he described various monotone transformations (continuous) of compact metric continua onto aposyndetic continua (these include continuous curves). Descriptions of Moore and Whyburn appear as special cases. However, transformations obtained in [4] did not leave certain aposyndetic continua invariant. In fact, some were mapped onto a point. In the present paper, a monotone transformation $T$ of an arbitrary compact metric continuum $M$ onto an aposyndetic continuum $A$ is described whose properties include that of leaving an aposyndetic continuum invariant. That is, if $M$ is aposyndetic, then $T(M)=M \sim A$. Thus, the elements of such an upper semi-continuous decomposition of $M$ (the sets $T^{-1}(x)$ for all $x$ in $A$ ) are "atomic." Results analogous to those in [4] are obtained for topological spaces. In fact, a description of an atomic decomposition of a connected topological space (not necessarily $T_{1}$ ) onto a connected aposyndetic Hausdorff space is given. If space is compact and metric, this description is equivalent to the one stated for a continuum in such a space.

It should be noted that the notion of a continuum being aposyndetic is a generalization of local connectedness and was introduced by F. B. Jones [1]. In a compact metric continuum, aposyndeticity is equivalent to semi-localconnectedness. A continuum, i.e., a closed and connected point set is said to be aposyndetic at a point $p$ with respect to a point $x$ provided that there exists a subcontinuum $N$ of $M-x$ and an open set 0 of $M$ such that $N \supset 0 \supset p$. If $M$ is aposyndetic at a point $p$ with respect to each point $x$ of $M-p$, then $M$ is said to be aposyndetic at $p$. Also, $M$ is aposyndetic if and only if $M$ is aposyndetic at each of its points.

The author wishes to express his gratitude to Professor F. B. Jones for having suggested these problems in classes and for his valuable encouragement.

Presented to the Society, November 23, 1956, received by the editors October 23, 1956. 
1. Description of atomic elements. The definition given here for the sets $M(p)$ is considerably less restrictive than that given for the sets $M(p)$ in [4]. A relation between these sets is shown in a later section. Note that nonseparated collections are no longer required but that the really useful properties (for this work) of nonseparated collections are still insisted upon.

Definition 1.1. Suppose that $M$ is a continuum, $p$ is a point of $M$, and that $Y$ is a point set property $\left({ }^{1}\right)$. Let $M(p)$ denote the set of all points $x$ in $M$ such that there does not exist a collection $K(p, x, Y)$ of closed separators $\left({ }^{2}\right)$ of $M$, each having Property $\mathrm{Y}$, such that

(1) there exists an element of $K$ which separates $p$ from $x$ in $M$ and

(2) for $g$ in $K$, a separation $M-g=A+B$, a continuum $N$ in $A$, and a point $b$ in $B$, there exist (i) a continuum $C$ containing an open set $D$ which contains $N$ and (ii) three disjoint elements $g_{1}, g_{2}$, and $g_{3}$ of $K$ each separating $C$ from $b$ in $M$ and $g_{2}$ separating $g_{1}$ from $g_{3}$ in $M$.

Although the following properties may be shown to hold for the sets $M(p)$ in quite general spaces, it will be assumed until later that $M$ is a compact metric continuum.

M1. For a point $p$ in $M$ and $y$ in $M(p), M(y)=M(p)$.

M2. For $p$ in $M, M(p)$ is closed.

M3. If $p$ and $x$ are points of $M$ such that $M(p) \neq M(x)$ and $K(p, x, Y)$ has Properties (1) and (2) of Definition 1.1, then (a) there exists an element of $K$ which separates $M(p)$ from $x$ in $M$ and (b) no element of $K$ separates two points of either $M(p)$ or $M(x)$ from each other in $M$.

M4. The collection $H$ of all sets $M(p)$ for the various points $p$ of $M$ is an upper semi-continuous collection $[4 ; 5]$ of disjoint closed sets such that $H^{*}$ (the logical sum of the elements of $H$ ) is $M$.

It will be shown later that for $p$ in $M, M(p)$ is connected provided that Property $\mathrm{Y}$ is that of being a point set. Here, compactness is required. Perhaps there is a short proof that $M(p)$ is connected. However, it is first shown that a component of a set $M(p)$ is an atomic element for the required decomposition of $M$ into an aposyndetic continuum. Then an argument easily follows which shows that $M(p)$ is identical to any one of its components.

2. Upper semi-continuous collections. As stated in [4], it is convenient to generalize the notion of an upper semi-continuous collection so that it can be used in a topological space which may fail to satisfy the first axiom of countability. When applied to collections of disjoint closed point sets in a compact metric space, this notion is equivalent to the usual definitions given by Moore [5] and Whyburn [7].

Let $U$ denote a collection of point sets in a topological space $T$. Here, a topological space is one defined as in either J. L. Kelley, General topology,

(1) Property Y may be the property of being a point set. On the other hand, it may be the property of being locally connected.

(2) A separator of $M$ is a subset $N$ of $M$ such that $N$ separates $M$ into two separated point sets, i.e., $M-N=H+K$ and $\bar{H} \cdot K=H \cdot \bar{K}=0$. 
1955, or Hall and Spencer, Elementary point set topology, 1956. Such an abstract space without the usual separation axiom is used merely to indicate the independence of some definitions from certain properties of topological spaces.

Definition 2.1. A subcollection $R$ of $U$ is said to be region in $U$ if and only if $R^{*}$ is an open set in $U^{*}$, i.e., open relative to $U^{*}$.

Definition 2.2. An element $g$ of $U$ is said to be a limit element of a subcollection $A$ of $U$ provided that every region $R$ in $U$ which contains $g$ also contains an element of $(A+g)-g$.

Definition 2.3. A collection $U$ of point sets is said to be upper semicontinuous provided that if (1) $A$ is a subcollection of $U$ and (2) $g$ and $h$ are two limit elements of $A$, then there exists a subcollection $B$ of $A$ such that $g$ is a limit element of $B$ and $h$ is not a limit element of $B$.

Suppose that $U$ denotes an upper semi-continuous collection of disjoint closed point sets such that $U^{*}=N$. Then the following statements are true.

U1. A necessary and sufficient condition that a subcollection $A$ of $U$ be closed in $U$ is that $A^{*}$ be closed in $N$.

U2. If $A$ is a subcollection of $U$ and $A^{*}$ is connected in $N$, then $A$ is connected in $U$.

3. Description of an upper semi-continuous decomposition $H$ of a compact metric continuum $M$ into an aposyndetic continuum. Suppose that Property $\mathrm{Y}$ is the property of being a point set. Then let $H$ denote the collection of all continua $C$ such that for some point $p$ in $M, C$ is a component of $M(p)$. Later, it is shown that $C=M(p)$.

Notation. The ordered pair $(U, R)$ denotes a hyperspace whose points are the elements of an upper semi-continuous collection $U$ and whose regions are those in the collection $R$ of all regions in $U$ defined by Definition 2.1.

Theorem 3.1. If Property $\mathrm{Y}$ is that of being a point set, then the collection $H$ is upper semi-continuous, and furthermore, the hyperspace $(H, R)$ is an aposyndetic compact metric continuum.

Proof. Since $M$ is compact and the collection of all sets $M(p)$ for the various points $p$ in $M$ is upper semi-continuous, it follows from the definition of $H$ that $H$ is upper semi-continuous. By a theorem [7, p. 123], $(H, R)$ is a compact metric continuum.

It remains to be shown that $(H, R)$ is aposyndetic. Suppose that $g$ and $h$ are two elements of $H$. There exists $p$ in $g$ and $x$ in $h$ such that $g$ and $h$ are components of $M(p)$ and $M(x)$, respectively. Either (1) $M(p) \neq M(x)$ or (2) $M(p)=M(x)$. Suppose that (1) is true. By Definition 1.1 and Property M3, there exists a collection $K(p, x, Y)$ which has Properties (1) and (2) of Definition 1.1 and an element of $K$ which separates $M(p)$ from $x$ in $M$. Also, by Definition 1.1, there exists a continuum $C$ containing an open set $D$ which contains $g$, an element $e$ of $K$, and a separation $M-e=S(C)+S(x)$ such that $S(C) \supset C$ and $S(x) \supset M(x)-e+x \supset h-e+x$. Let $H(C)$ denote the collection 
of all elements in $H$ which intersect $C$. From Property U2, it follows that $H(C)$ is a continuum in the hyperspace $(H, R)$. Also, $H(C)$ contains an open set in $H$ which contains $g$ and $H(C) D h$. Therefore, $(H, R)$ is aposyndetic at $g$ with respect to $h$.

Next, consider the case that $M(p)=M(x)$ is true. Since $M(p) \supset g+h$, $M(p)$ is not connected. Thus, $M(p)$ is the sum of two disjoint closed sets $A$ and $B$ containing $g$ and $h$, respectively. There exists a closed set $E$ which separates $A$ from $B$ in $M$. Now, let $H(E)$ denote the set of all elements in $H$ which intersect $E$. Thus, $H(E)$ separates $g$ from $h$ in $H$. Furthermore, no element of $H(E)$ is a component of $M(p)$. By an argument analogous to one given for case (1), $(H, R)$ is aposyndetic at each point of $H(E)$ with respect to $g+h$. That is, for $k$ in $H(E)$, there exists a continuum $F$ in $H-(g+h)$ containing an open set 0 in $H$ which contains $k$. Since $H(E)$ is closed and compact, there exists a positive integer $n$ and such continua $F_{i}$ in $H, 1 \leqq i \leqq n$, such that $\sum_{i=1}^{n} F_{i}$ separates $g$ from $h$ in $H$. Let $H-\sum_{i=1}^{n} F_{i}=S(g)+S(h)$ denote this separation. Now, in $S(g)+\sum_{i-1}^{n} F_{i}$ there exists a continuum $Q$ in $H-h$ which contains an open set 0 in $H$ which contains $g$. Consequently, $(H, R)$ is aposyndetic at $g$ with respect to $h$. It follows that $(H, R)$ is aposyndetic.

4. Existence theorem. Once it is shown that the description given in $\S 3$ of the decomposition $H$ of a continuum $M$ into an aposyndetic continuum leaves an arbitrary aposyndetic continuum invariant (i.e., each element of $H$ is a point of $M$ ), then it follows that an atomic decomposition has been described. Another consequence would be that the sets $M(p)$ are continua.

THEOREM 4.1. If $M$ is a compact metric aposyndetic continuum and $p$ is a point of $M$, then $M(p)=p$ provided that Property $\mathrm{Y}$ is that of being a point set.

Proof. For each proper subcontinuum $N$ of $M$ and each point $x$ of $M-N$, there exists a continuous mapping $f$ of $M$ onto the closed interval $[0,1]$ such that $f(N)=0, f(x)=1$, and for $y$ in $M-(N+x), 0<f(y)<1$. Associate exactly one such mapping $f$ with the pair $(N, x)$. Now, let $K(p, x, Y)$ denote the collection of all sets $f^{-1}(z)$ where $0<z<1$ for each triple $(N, x, f)$ described above.

Next, it is shown that $K(p, x, Y)$ has the desired Properties (1) and (2) of Definition 1.1. Let $x$ denote a point of $M-p$. Certainly, $K$ has Property (1). Now, let $M-g=A+B$ denote a separation of $M$ by an element $g$ in $K, N$ a continuum in $A$, and $b$ a point of $B$. Since $M$ is aposyndetic at each point of $N$ with respect to $b$, it follows that there exists a continuum $C$ in $M-b$ containing an open set which contains $N$. By definition of $(N, b, f)$, there exists $z, 0<z<1$, such that $f^{-1}(z)$ separates $C$ from $b$ in $M$. Hence, it is assured that $K(p, x, Y)$ has Property (2) of Definition 1.1. Consequently, $M(p)=p$.

Now, Theorem 4.2 readily follows. 
THEOREM 4.2. If $M$ is a compact metric aposyndetic continuum, then the hyperspace $(K, R)$ is identical to $M$ provided that Property $\mathrm{Y}$ is that of being a point set.

By use of Theorems 3.1, 4.1, and 4.2, a proof of Theorem 4.3 may be obtained.

THEOREM 4.3. If $M$ is a compact metric continuum, then $M(p)$ is a continuum for each point $p$ in $M$ provided that Property $\mathrm{Y}$ is that of being a point set.

Now, one may observe that the sets $M(p)$ are atomic with this definition of Property $\mathrm{Y}$ and with respect to the property that the hyperspace $(K, R)$ be aposyndetic.

5. Sets $M(p)$ related to sets $M(p)$ in [4]. A set $M(p)$ defined by Definition 1.1 is a subset of a set $M(p)$ defined in [4] using the same Property Y in each case. Example 6.1 of [4] shows that the sets $M(p)$ defined there are not necessarily connected. Certain subsets $S(g, x)$ of these are defined which are components of the sets $M(p)$ in the compact case. In [4], the essential restriction on $M(p, x, Y)$ was that it be an uncountable nonseparated collection of disjoint separators of $M$. This may be accomplished by two rather natural restrictions on $K(p, x, Y)$ in Definition 1.1. Consider the following.

Definition 5.1 (Definition 1.1 plus restrictions on $K$ ). Suppose that $M$ is a continuum, $p$ is a point of $M$, and $Y$ is a point set property. Let $M(p)$ denote the set of all points $x$ in $M$ such that there does not exist a collection $K(p, x, Y)$ of irreducible $\left(^{3}\right)$ separators of $M$, each having Property $Y$, such that

(1) there exists an element of $G$ which separates $p$ from $x$ in $M$ and

(2) for $g$ in $K$, a separation $M-g=A+B$, a continuum $N$ in $A$, and a continuum $Q$ in $B$, there exist (i) a continuum $C$ containing an open set $D$ which contains $N$ and (ii) three disjoint elements $g_{1}, g_{2}$, and $g_{3}$ of $K$ such that each separates $C$ from $Q$ in $M$ and $g_{2}$ separates $g_{1}$ from $g_{3}$ in $M$.

TheOREM 5.2. Suppose that $M$ is a continuum in a topological space. Then the sets $M(p)$ defined by Definition 5.1 are identical with the sets $M(p)$ in [4].

Proof. First, establish the following lemma.

Lemma 5.3. If $a, b$, and $c$ are irreducible separators of $M$ and $b$ separates $a$ from $c$ in $M$, then the collection consisting of $a, b$, and $c$ is nonseparated $\left({ }^{4}\right)$. $M$.

(3) A separator $A$ of $M$ is said to be irreducible if and only if no proper subset of $A$ separates

(4) A collection $C$ of subsets of $M$ will be called nonseparated if and only if (1) the elements of $C$ are disjoint and (2) no element of $C$ separates in $M$ two points belonging to any other one element of $C$ [cf. $7 ; 9]$. 
Proof of lemma. Consider a separation $M-b=S(a)+S(c)$. If $b$ separates a point $x$ of $c$ from a point $y$ of $c$, then $S(c)=S(x)+S(y)$ the sum of two separated sets containing $x$ and $y$, respectively. There exists a separation $M-c=P+Q$ where $P \supset S(a)+b$. Consequently, $Q=S(x)+S(y)-c$. Here, $Q+c$ is not connected. Thus, some proper subset of $c$ separates $M$. This is impossible since $c$ is an irreducible separator of $M$.

Since both $S(a)+b$ and $S(c)+b$ are connected, it follows that the collection consisting of $a, b$, and $c$ is nonseparated.

Proof of Theorem 5.2. Let $p$ and $x$ denote points of $M$ such that $M(p) D x$ (Definition 5.1). By Definition 5.1, there exist three disjoint irreducible separators $a, b$, and $c$ such that each separates $p$ from $x$ in $M$ and $b$ separates $a$ from $c$ in $M$. By Lemma 5.3, the collection consisting of $a, b$, and $c$ is nonseparated. It is to be shown that there exists an uncountable nonseparated collection $M(p, x, Y)$ of disjoint separators of $M$ each of which separates $p$ from $x$ in $M$.

Now, there exists a separation $M-b=S(a)+S(c)$ such that $S(a) \supset p+a$ and $S(c) \supset c+x$. Since $a$ and $c$ are irreducible separators of $M$, there exist subcontinua $N_{a}$ and $N_{c}$ of $S(a)$ and $S(c)$ containing $p+a$ and $c+x$, respectively. Again, by Definition 5.1, there exist three disjoint irreducible separators $d, e$, and $f$ of $M$ each separating $N_{a}$ from $N_{c}$ in $M$ and $e$ separating $d$ from $f$ in $M$. Here, $a, d, e, f$, and $c$ form a nonseparated collection which is naturally ordered $\left.{ }^{5}\right)$. Continuing in such a way, it is possible to obtain an infinite nonseparated collection $K_{1}$ of disjoint irreducible separators of $M$ such that (1) each element of $K_{1}$ separates $p$ from $x$ in $M$, (2) for each pair of elements $g$ and $h$ of $K_{1}$, there exists an element of $K_{1}$ which separates $p+g$ from $h+x$ in $M$, and (3) $K_{1}$ is naturally ordered. Consequently, there exists a continuous mapping $f$ of $M$ onto $[0,1]$ such that $f(p)=0 ; f(x)=1$; for $g$ in $K_{1}, 0<f(g)<1$; and for $g$ in $K_{1}, f^{-1} f(g)=g$. There exist uncountably many numbers $y$ between 0 and 1 which are limit points of sequences $\left[f\left(g_{l i}\right)\right]$ and $\left[f\left(g_{r i}\right)\right]$ where $g_{l i}$ and $g_{r i}$ are elements of $K_{1}, f\left(g_{l i}\right)<f\left(g_{l i+1}\right)$, and $f\left(g_{r i}\right)>f\left(g_{r i+1}\right)$, for each $i$. Now, $f^{-1}(y)$ is a closed set which separates $M$ in to exactly two sets one containing $p$ and the other containing $x$. Furthermore, if $K_{2}$ denotes the collection of all these sets $f^{-1}(y)$ plus the elements of $K_{1}$, then $K_{2}$ is a nonseparated collection of disjoint separators of $M$. Thus, by the definition of the sets $M(p)$ in [4], $M(p) D x$.

The truth of Theorem 5.2 is now easy to establish.

The following example shows that the additions to Definition 1.1 in Definition 5.1 are both necessary for the validity of Theorem 5.2.

${ }^{(5)}$ A set of collection of sets $N$ is said to be ordered or to possess an order provided that a definition of "preceding" (indicated <) can be defined for each pair of elements which is asymmetric and transitive. Suppose that for $x \in N, P_{x}$ denotes the logical sum of all elements of $N$ preceding $x$ and $F_{x}$ the sum of all those preceded by $x$ (or following $x$ ). Then the order in $N$ is said to be a natural order if and only if for each $x \in N, \bar{P}_{x} \cdot F_{x}=P_{x} \cdot \bar{F}_{x}=0$, cf. $[7 ; 9]$. 
Example 5.4. Let Figure 1 suffice to describe a continuum $M$ in the number plane. Suppose that Property $\mathrm{Y}$ is that of being an irreducible separator of $M$. With $p$ and $x$ as indicated in Figure 1, it follows that $M(p) \supset x$. Here, Definition 1.1 is used.

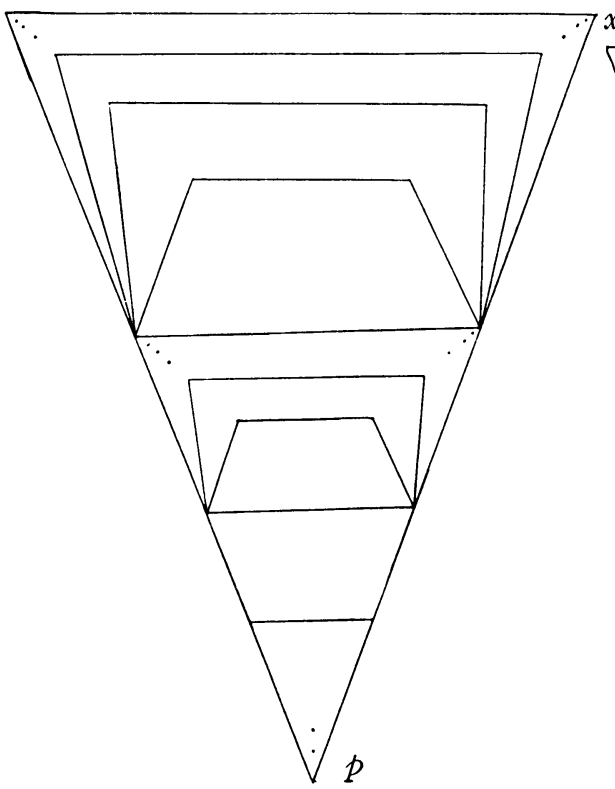

FIG. 1

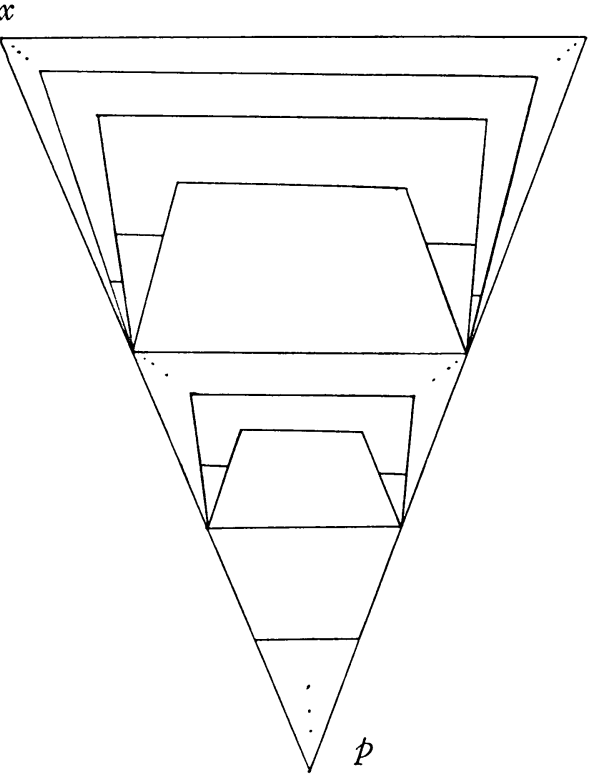

FIG. 2

ExAmple 5.5. Let Figure 2 suffice to describe a continuum $M$ in the number plane. Suppose that $p$ and $x$ are as indicated there. Then it follows by Definition 1.1 that $M(p) \triangleright x$; but by Definition 2.1 of [4], $M(p) \supset x$. Thus, Examples 5.4 and 5.5 show that both restrictions added to Definition 1.1 in Definition 5.1 are necessary for the validity of Theorem 5.2.

6. Sets $S(g, x)$. As seen in an earlier section, the sets $M(p)$ are continua provided that $M$ is a compact metric continuum and Property $\mathrm{Y}$ is that of being a point set. However, if Property $\mathrm{Y}$ is that of being an irreducible separator of $M$, then $M(p)$ may be nontrivially totally disconnected even though $M$ is a compact metric continuum. Example 6.1 of [4] is such an example. Here, there is an $M(p)$ defined by any one of the three definitions given in this paper and in [4] with the above-mentioned Property Y such that $M(p)$ is not connected. Consequently, it is convenient to consider certain subsets $S(g, x)$ of the sets $M(p)=g$. These are used to obtain an upper semi-continuous decomposition of a continuum into an aposyndetic continuum.

Now, define the sets $S(g, x)$.

Definition 6.1. Suppose that $M$ is a continuum and that $H$ denotes the collection of all point sets $M(p)$ for the various points $p$ in $M$ where $M(p)$ is 
defined by either of the definitions given in this paper [Definitions 1.1, 5.1, and 8.1] or by Definition 2.1 of [4]. Then, for $x \in g \in H$, let $S(g, x)$ denote the set of all points $y$ of $g$ such that there does not exist a subcollection $C$ of $H$ such that (1) $C^{*}$ is the sum of a finite number of continua and (2) $C^{*}$ separates $x$ from $y$ in $M$.

The sets $S(g, x)$ have the following properties.

S1. For $g$ in $H$ and $x$ in $g, S(g, x)$ is a closed point set.

S2. If $x$ and $y$ denote points of $g$ in $H$ and $S(g, x) \cdot S(g, y) \neq 0$, then $S(g, x)$ $=S(g, y)$.

Using methods described in this paper and in [4], the following theorems may be proved for a compact metric continuum $M$.

Theorem 6.2. If $x \in g \in H$, then $S(g, x)$ is the component of $g$ which contains $x$.

Theorem 6.3. The collection $G$ [of all sets $S(g, x)$ for the various elements $g$ in $H$ and the various points $x$ in $g]$ is an upper semi-continuous collection of disjoint closed point sets filling up $M$; and furthermore, $(G, R)$ is a compact aposyndetic metric continuum.

7. Consequences of some other definitions of Property Y. Definitions of Property Y may, of course, vary from that of being a point set to rather restrictive properties such as that of being locally connected. In the latter case, it was shown in [4] that the hyperspace is a continuous curve. An analogous result is obtained here. Also, if Property $\mathrm{Y}$ is that of being a point, the hyperspace is an acyclic continuous curve. Thus, the results of Moore $[5 ; 6]$ and Whyburn $[7 ; 8 ; 9]$ concerning such decompositions are obtained by a seemingly weaker description of an upper semi-continuous decomposition [cf. 4, Corollary 6.51, p. 90].

The following theorems may be proved by methods used here and in [4].

Theorem 7.1. Suppose that $M$ is a compact metric continuum and that Property $\mathrm{Y}$ is that of being locally connected. Then the hyperspace $(G, R)$ is a compact metric continuous curve.

Theorem 7.2. Suppose that $M$ is a compact metric continuum and that Property $\mathrm{Y}$ is that of being exactly one point. Then $(G, R)$ is a compact metric acyclic continuous curve.

TheOREm 7.3. Suppose that $M$ is a compact subcontinuum of the number plane and that Property $\mathrm{Y}$ is that of being the sum of a finite number of continua. Then $(G, R)$ is a compact metric continuous curve.

8. An atomic decomposition of a connected topological space. By a natural alteration of Definition 1.1, one may obtain a decomposition of a connected topological space $M$ (not necessarily a $T_{1}$ space) into an aposyndetic connected Hausdorff space. In case $M$ is a compact metric continuum, the 
sets $M(p)$ given by this alteration are identical to those defined in Definition 1.1 where Property $\mathrm{Y}$ is that of being a point set. This is certainly a desirable property. Results analogous to those in [4] but much more general are obtained. With a suitable definition of Property Y, the sets $M(p)$ defined below are "atomic" with respect to the property that the hyperspace be an aposyndetic continuum.

Definition 8.1. Suppose that $p$ is a point of a continuum $M$ and that $Y$ is a point set property. Let $M(p)$ denote the set of all points $x$ in $M$ such that there does not exist a collection $K(p, x, Y)$ of separators of $M$, each having Property $\mathrm{Y}$, such that

(1) there exists an element of $K$ which separates $p$ from $x$ in $M$ and

(2) for a separation $M-g=A+B$, a point $a$ in $A$ and a point $b$ in $B$, there exist (i) open sets $A_{1}$ and $B_{1}$ containing $a$ and $b$, respectively, (ii) subcontinua $C$ and $D$ of $M$ such that $M-B_{1} \supset C \supset A_{1}$ and $M-A_{1} \supset D \supset B_{1}$, (iii) an element $g$ of $K$ such that $g$ separates $A_{1}$ from $B_{1}$ in $M$, and (iv) for a point $a_{1}$ in $A_{1}$ and a point $y$ in $M-A_{1}$, there exist open sets $A_{2}$ and $B_{2}$ containing $a_{1}$ and $y$, respectively, subcontinua $C_{1}$ and $D_{1}$ of $M$ such that $M-B_{2}$ $\supset C_{1} \supset A_{2}$ and $M-A_{2} \supset D_{1} \supset B_{2}$, and an element $k$ of $K$ such that $k$ separates $A_{2}$ from $B_{2}$ in $M$.

It easily follows that the sets $M(p)$ defined above have the properties listed below [cf. Properties M1-M4].

K1. For a point $p$ in $M$ and $y$ in $M(p), M(y)=M(p)$.

$\mathrm{K} 2$. For $p$ in $M, M(p)$ is closed.

K3. If $p$ and $x$ are points of $M$ such that $M(p) \neq M(x)$ and $K(p, x, Y)$ has Properties (1) and (2) of Definition 8.1, then there exist an element $g$ of $K$, continua $C$ and $D$, and open sets $A_{1}$ and $B_{1}$ such that (a) $M-B_{1} \supset C \supset A_{1}$ $\supset M(p)$ and $M-A_{1} \supset D \supset B_{1} \supset M(x)$, (b) $g$ separates $A_{1}$ from $B_{1}$ in $M$, and (c) $g, A_{1}, B_{1}, C$, and $D$ have Property (2) of Definition 8.1.

In Property K4 below, the notion of upper semi-continuity described by Definitions $2.1-2.3$ is used. Note that the continuum $M$ may fail to satisfy the first axiom of countability as well as the Hausdorff separation axiom.

$\mathrm{K} 4$. The collection $H$ of all sets $M(p)$ for the various points $p$ of $M$ is an upper semi-continuous collection of disjoint closed sets such that $H^{*}=M$.

Proof of Property K4. Let $M(p)$ and $M(x)$ denote two elements of $H$. There exist $g, A_{1}$, and $B_{1}$ having the properties attributed to them in $\mathrm{K} 3$. The collections $H\left(A_{1}\right)$ and $H\left(B_{1}\right)$ of all elements of $H$ which intersect $A_{1}$ and $B_{1}$, respectively, are disjoint regions in $H$, that is, $H\left(A_{1}\right)^{*}$ and $H\left(B_{1}\right)^{*}$ are disjoint open sets in $M$. Consequently, the validity of Property $\mathrm{K} 4 \mathrm{fol}$ lows.

Consider the next theorem of decomposition.

Theorem 8.2. Suppose that $M$ is a connected topological space and that $H$ is the collection of all sets $M(p)$ [Definition 8.2] for the various points $p$ of $M$. Then $(H, R)$ is an aposyndetic connected Hausdorff space. 
Proof. From Property $\mathrm{K} 4$, it easily follows that $(H, R)$ is a Hausdorff space. It is connected by Property U2 of $\S 2$.

In order to show that $(H, R)$ is aposyndetic, use the notation given in the proof of Property K4. Thus, there exists a subcontinuum $C$ of $M$ containing an open set $A_{1}$ such that $M-C$ contains an open set $B_{1}$ containing $M(x)$ (where $M(p)$ and $M(x)$ are any two elements of $H$ ) such that $H\left(A_{1}\right)$ and $H\left(B_{1}\right)$ are disjoint open sets in $(H, R)$. Now, let $H(C)$ denote the collection of all elements in $H$ which intersect $C$. It follows that the closure $\bar{H}(C)$ of $H(C)$ in $(H, R)$ is a subcontinuum of $H-M(x)$ which contains an open set in $H$ which contains $M(p)$. Hence, $(H, R)$ is aposyndetic.

One may wish to use various definitions of Property $\mathrm{Y}$ in such a space $M$ and consequently the sets $S(g, x)$ as defined earlier. Definition 6.1 which defines these sets applies here. By the methods of this paper and of [4], the following theorem may be proved.

THEOREM 8.3. Suppose that $M$ is a connected topological space and that $G$ is the collection of all sets $S(g, x)$ for the various elements $g$ in $H$ and the various points $x$ in $g$. Then $G$ is an upper semi-continuous collection of disjoint closed sets filling up $M$; and furthermore, $(G, R)$ is an aposyndetic connected Hausdorff space.

The next theorem yields the desired atomic decomposition.

Theorem 8.4. If Property $\mathrm{Y}$ is that of being a point set and $M$ is a connected aposyndetic topological space, then $(H, R)$ is identical with $M$, that is, for a point $p$ in $M, M(p)=p$.

Proof. Let $p$ and $x$ denote two points of $M$. Also, let $y$ and $z$ denote any two points of $M$. Since $M$ is aposyndetic, there exist subcontinua $C$ and $D$ of $M$ and open sets $A_{1}$ and $B_{1}$ such that $M-B_{1} \supset C \supset A_{1} \supset y$ and $M-A_{1} \supset D \supset B_{1} \supset z$. Associate with $y$ and $z$ the sets $\bar{B}_{1}-B_{1}$ and $\bar{A}_{1}-A_{1}$, respectively. Let $K(p, x, Y)$ denote the collection of all sets $\bar{B}_{1}-B_{1}$ and $\bar{A}_{1}-A_{1}$ associated with $y$ and $z$ for all the various pairs of two points $y$ and $z$ in $M$.

It follows that $M(p)=p$.

9. Concluding remarks. In the compact metric case, the existence of a continuous monotone mapping $f$ of an arbitrary continuum onto an aposyndetic continuum has been shown. A similar result has been given for the noncompact case but here the mapping may fail to be monotone.

Now that an atomic decomposition of continua $M$ into aposyndetic continua $A$ is possible, questions naturally arise concerning consequences of definitions of Property $\mathrm{Y}$ and the sets $M(p)$. For example, what must be known about the sets $M(p)$ (inverse sets of a continuous mapping) in order to conclude that $M$ has the fixed point property? It is easy to state a result which gives hope that a general solution to this problem is possible. Suppose that $T$ is a continuous mapping of a continuum $M$ onto an acyclic continuous 
curve $C$ such that for $x$ in $C, T^{-1}(x)=M(p), p$ in $M$, has the fixed point property. Then $M$ has the fixed point property under homeomorphisms of $M$ onto $M$. Here, one applies G. T. Whyburn's cyclic element theory [7]. Thus, one asks the following question. What are necessary and sufficient conditions that the sets $M(p)$ have the fixed point property? Some progress has been made in these and other directions.

\section{BIBLIOGRAPHY}

1. F. Burton Jones, Aposyndetic continua and certain boundary problems, Amer. J. Math. vol. 63 (1941) pp. 545-553.

2. - Concerning aposyndetic and non-aposyndetic continua, Bull. Amer. Math. Soc. vol. 58 (1952) pp. 137-151.

3. R. G. Lubben, Concerning the decomposition and amalgamation of points, upper semicontinuous collections, and topological extensions, Trans. Amer. Math. Soc. vol. 49 (1941) pp. $410-466$.

4. Louis F. McAuley, On decomposition of continua into aposyndetic continua, Trans. Amer. Math. Soc. vol. 81 (1956) pp. 74-91.

5. R. L. Moore, Foundations of point set theory, Amer. Math. Soc. Colloquium Publications, vol. 13, New York, American Mathematical Society, 1932.

6. - Fundamental theorems concerning point sets, The Rice Institute Pamphlet vol. 23 (1936) pp. 1-74.

7. G. T. Whyburn, Analytic topology, Amer. Math. Soc. Colloquium Publications, vol. 28, New York, American Mathematical Society, 1942.

8. - Concerning collections of cuttings of connected point sets, Bull. Amer. Math. Soc. vol. 35 (1929) pp. 87-104.

9. - Non-separated cuttings of connected point sets, Trans. Amer. Math. Soc. vol. 33 (1931) pp. 444-454.

10. —, Semi-locally-connected sets, Amer. J. Math. vol. 61 (1939) pp. 733-749.

The University of Wisconsin, Madison, Wis. 